\title{
Analyzing Black Hole Super-Radiance Emission of Particles/Energy from a Black Hole as a Gedankenexperiment to Get Bounds on the Mass of a Graviton
}

\author{
A. Beckwith \\ Department of Physics, Chongqing University, Chongqing 400044, China \\ Correspondence should be addressed to A. Beckwith; rwill9955b@gmail.com
}

Received 18 December 2013; Revised 10 February 2014; Accepted 10 February 2014; Published 3 April 2014

Academic Editor: Christian Corda

Copyright (C) 2014 A. Beckwith. This is an open access article distributed under the Creative Commons Attribution License, which permits unrestricted use, distribution, and reproduction in any medium, provided the original work is properly cited. The publication of this article was funded by $\mathrm{SCOAP}^{3}$.

Use of super-radiance in $\mathrm{BH}$ physics, so $d E / d t<0$ specifies conditions for a mass of a graviton being less than or equal to $10^{65}$ grams, allows for determing what role additional dimensions may play in removing the datum that massive gravitons lead to 3/4th the bending of light past the planet Mercury. The present document makes a given differentiation between super-radiance in the case of conventional BHs and Braneworld BH super-radiance, which may delineate whether Braneworlds contribute to an admissible massive graviton in terms of removing the usual problem of the 3/4th the bending of light past the planet Mercury which is normally associated with massive gravitons. This leads to a fork in the road between two alternatives with the possibility of needing a multiverse containment of $\mathrm{BH}$ structure or embracing what Hawkings wrote up recently, namely, a redo of the event horizon hypothesis as we know it.

\section{Introduction: Massive Gravity and How to Get It to Commensurate with Black Hole Physics}

We are now attempting to come up with criteria for either massless or massive gravitons. Our preferred way to do it distinguishing between the two forms of super-radiance. One built about Kerr black holes [1], and the other involving brane theory [2]; with the brane theory version of superradiance, perhaps correcting a problem as to when a massive graviton would, without brane theory, lead to $3 / 4$ th the angular bending of light and be seen experimentally. We briefly allude to both of these cases in the introduction below, before giving more details to this phenomenon in Sections 2 and 3 .

In general, relativity of the metric $g_{a b}(x, t)$ is a set of numbers associated with each point which gives the distance to neighboring points. That is, general relativity is a classical theory. As it is designated by GR traditionalists [3], the graviton is usually stated to be massless, with two spin states and with two polarizations. Adding a mass to the graviton results in 5 polarizations plus other problems [ 4 , 5]; that is, in [4], there is a description of how a massive graviton leads to $3 / 4$ th the calculated bending of light pass the mass of Mercury, as seen in the 1919 experiment. Reference [5] has details on the five polarization states, which are another problem. One cannot go from a massive graviton and eliminate mass from the graviton and then neatly recover the easier spin dynamics (2 polarization states) and vastly simpler situation where one has recovered the Schwartzshield metric. As [5] discusses, in its page 92, that this easy recovery of the Schwartzshield metric, if a graviton mass goes to zero, is impossible. Also note that note [4] has a discussion on how the bending of light is not commensurate with GR for massive graviton, which is equivalent to a discussion on a phenomenological ghost state for the trace of $h$, which is given by [6] and occurs regardless of whether the mass for graviton nearly goes to zero. In [7], Csáki et al., have given a temporary fix to restore the bending of light for massive gravitons and to remove the 3/4th angle deflection from the 1919 GR test value, and this is by the use of brane theory. What this document will do will be to try to establish massive 
gravitons as super-radiant emission candidates from black holes [8] and, in doing so, provide another framework for their analysis which would embed them in GR. In doing so, one should keep in mind that this is a thought experiment and that the author is fully aware of how hard it would be to perform experimental measurements. In coming up with criteria as to graviton mass, we are also, by extension, considering the Myers-Perry higher dimensional model of black holes [9] and commenting upon its applications, some of which are in [10] and all of which start with the implications of $d E / d t<0$, leading to "leakage" from a black hole. That is, energy of the black hole "decreases" in time. That is, there are ghost states, where $h$ is the trace of $h(i, j)$ which is a GW perturbation of the flat Euclidian metric, a possibility that brane theory and higher dimensions may remove the 3/4th angle of bent light calculated for massive gravitons, and a suitable thought experiment as given below may allow for $d E / d t$ allowing us to determine whether higher dimensional models are justifiable. This is the reason why the superradiance phenomenology is being investigated, that is, of bending of angle of light divergence from GR models using massive Gravitons. Does $d E / d t<0$ imply that there are brane theory states which may remove the 3/4th bending of light divergence from GR by massive gravitons? And can a superradiance model for when $d E / d t<0$ imply conditions for which brane worlds have to be considered [2], as opposed to the simpler model proposed by Padmanabhan [1].

The paper will differentiate between Kerr BH [1] versions of super-radiance and brane theory $\mathrm{BH}$ super-radiance [2] and, secondly, afterwards, inquire about whether a $\mathrm{BH}$ in brane theory configuration is satisfied, if the simper Kerr $\mathrm{BH}$ super-radiance criteria is not satisfied. After these two versions are distinguished, we will then discuss experimental criteria which may result in determining whther Kerr $\mathrm{BH}$ super-radiance occurs [1] or brane theory super-radiance occurs [2]; if only Kerr $\mathrm{BH}$ super-radiance occurs, the likelihood of massive Gravitons is remote. If brane theory $\mathrm{BH}$ super-radiance occurs, then there may [2] be conditions for which the 3/4th error in light bending is removed, permitting massive Gravitons.

\section{What is Super-Radiance in Black Hole Physics? First: The Padmanabhan Treatment for Kerr BHs}

We, first of all, consider a simplified version of superradiance. In simple language, super-radiance involves having incoming radiation scattered off the horizon of a $\mathrm{BH}$ and radiated outward, so the net flow of energy is $d E / d t<0$ radiation energy with a frequency bounded by $0<\omega<$ $m \cdot \Omega_{H}$ [1]. In this case $m$ is a quantum number, the frequency $\omega$ is for radiation infalling to the event horizon of the $\mathrm{BH}$, and the term $\Omega_{H}$ is the angular velocity of a KERR black hole [2]. This paper, first of all, examines Padmanabhan's derivation of super-radiance [2] stating its application to the graviton, with mass, and making then a referral to the likelihood of measurement which ties in with the metric $g_{\mu \nu}$ being perturbed from flat space values by $h_{00}, h_{0 i}$, and $h_{i j}$ [7], thereby making the case, due to the mass dependence of the black hole, that super-radiance would almost certainly not be observable but would firmly embed massive gravitons in GR in spite of the view point offered in [3]. Doing so would mean that [1] has the following formulation; with respect to when $d E / d t<0$, which occurs for super radiance; in (1) below; we set $\mathrm{cl}$ as a constant, radiation frequency omega as the frequency of radiation approaching a black hole, the number $m$ as a quantum number, and a definite given value for the angular velocity of a black hole. Here, after the Padmanabhan derivation of what $d E / d t<0$ means, there will be a separate, brane theory derivation of $\mathrm{BH}$ super-radiance [2] which has provisionally $0<\omega<\sum_{J=1}^{N / 2} m_{J} \cdot\left(\Omega_{H}\right)_{J}$ [2], where $N$ is the number of dimensions. The 2 nd frequency dependence for when $N$ can go up to at least 10 or so will be remarked after we finish the Padmanabhan frequency dependence for super-radiance, as given below for a "classical" Kerr BH. To initiate our analysis of the physics happening in due to [1], we formulate super-radiance by (1) given below

$$
\frac{d E}{d t}=c_{1} \cdot \omega \cdot\left[\omega-m \cdot \Omega_{H}\right] .
$$

In this case, according to Padmanabhan, $c_{1}$ is a constant, which is defined via writing (1) via [1]. Consider

$$
\begin{aligned}
\frac{d E}{d t}= & \frac{M \cdot r_{H}}{2 \pi} \cdot\left[\int\left(S^{2}(\theta) \cdot \sin ^{2} \theta \cdot d \theta\right) \cdot d \phi\right] \\
& \cdot \omega \cdot\left[\omega-m \cdot \Omega_{H}\right] \\
= & \text { flux-of-energy-through-horizon } \\
\Longleftrightarrow & c_{1} \equiv \frac{M \cdot r_{H}}{2 \pi} \cdot\left[\int\left(S^{2}(\theta) \cdot \sin ^{2} \theta \cdot d \theta\right) \cdot d \phi\right]=\text { const., }
\end{aligned}
$$

where, for a massless scalar field, one has the function $S$ for a "surface area" function, defined as follows [1]:

$$
\begin{aligned}
\exists S^{2}(\theta) & \Longleftrightarrow(-g)^{-1 / 2} \partial_{b}\left[(-g)^{-1 / 2} \cdot g^{a b} \cdot \partial_{a} \Phi\right]=0 \\
& \Longleftrightarrow \Phi \equiv e^{-i \omega t} e^{i m \cdot \phi} \cdot R(r) \cdot S(\theta) .
\end{aligned}
$$

In this case, mass $M$ is for the source, that is, later for the mass $M$ of a GW generator, in this case a $\mathrm{BH}$. Also, here, $r_{H}$ is the horizon radius, as specified. And this will have its application to the issue of gravitons of a small mass spirialing into a $\mathrm{BH}$, with the $\mathrm{BH}$ subsequently releasing radiation via $d E / d t<0$, with the given versions of a $\mathrm{BH}$ set of parameters [1] for a KERR BH. The following (4) comes as far as angular velocity of the $\mathrm{BH}$, as well as the following sets of parameters. Here, the phenomenon of super-radiance is impossible, if (6) below is zero. More on this point about when super-radiance is impossible will be discussed later in the text. Consider

$$
\begin{gathered}
\Omega_{H}=\frac{a}{2 M_{\mathrm{BH}} \cdot r_{H}}, \\
r_{H}=M_{\mathrm{BH}}-\sqrt{{M_{\mathrm{BH}}^{2}-a^{2}}^{2}} \\
a=\sqrt{x^{2}+y^{2}} .
\end{gathered}
$$


Then,

$$
0<\frac{\omega}{m}<\Omega_{H}
$$

Note that there are conditions, based upon (4) above, which go to zero, due to the numerator, in a manner which means when there is no angular velocity for the black hole; that the frequency of the incoming radiation is set equal to zero and that there is, effectively no super-radiance. This will obviously lead to the classical description of $\mathrm{BH}$ physics. A problem, though, is that, recently, Hawkings has stated that not all is well in $\mathrm{BH}$ event horizons and that scrambled information could possibly leave a $\mathrm{BH}$, in opposition.

2.1. Examining Super-Radiance When There Is More than 4 Dimensions as to BH Physics. As said before [2],

$$
0<\omega<\sum_{J=1}^{N / 2} m_{J} \cdot\left(\Omega_{H}\right)_{J}
$$

In doing so, $N$ as given above is a measure of dimensions as to the $\mathrm{BH}$, and the difference in this from (7) in part is also due to

$$
\begin{aligned}
\Omega_{H} & =\left.\frac{a}{2 M_{\mathrm{BH}} \cdot r_{H}}\right|_{\text {Kerr-BH }} \\
& \left.\underset{\text { Kerr-BH } \rightarrow \text { Myers-Perry-BH }}{\longrightarrow} \frac{a}{a^{2}+r_{H}^{2}}\right|_{\text {Myers-Perry-BH }} .
\end{aligned}
$$

The numerator of the above is still defined by the square root of $x^{2}+y^{2}$ and could go to zero for certain quantum numbers, $m_{J}$, and we would then paraphrase the right hand side of (9) as functionally being

$$
\Omega_{j}=\frac{a_{J}}{a_{J}^{2}+r_{H}^{2}}
$$

as frequency of $\mathrm{BH}$ arises due to the jth component of $\mathrm{BH}$ angular Momentum $J_{j}$.

So, then, one has a rewrite of (8) as given, with a slightly different angular frequency for BHs as by [2],

$$
0<\omega<\left.\sum_{j=1}^{N / 2} m_{j} \frac{a_{j}}{a_{j}^{2}+r_{H}^{2}}\right|_{\text {Myers-Perry-BH }} .
$$

This is to be compared with the Padmanabhan version of super-radiance as given by [1]:

$$
0<\omega<\left.m \cdot \frac{a}{2 M_{\mathrm{BH}} \cdot r_{H}}\right|_{\text {Kerr-BH }} .
$$

We will be commenting upon what the experimental signatures of both (11) and (12) could be and why in the next section.

\section{Could Super-Radiance Be Observed Experimentally, and What Good Is This Thought Experiment?}

Super-radiance is really about the same as particle production from a $\mathrm{BH}$. From Padmanabhan [1] is a vital result which is given in the following quote.

If we think of super-radiance as stimulated emission of radiation by the black hole in certain modes, owing the presnce [sic] of the incoming wave, it seems natural to expect spontaneous emission of radiation in various modes by the black hole in quantum field theory. The black hole evaporation (then) can be thought of as spontaneous emission of particles that survives even in the limit of zero angular momentum of the black hole.

Furthermore, on the same page, page 623 of [1] states the following.

It seems natural to assume that this source of energy radiated to infinity is the mass of the collapsing structure.

Leading to Formula 14.143 of [1] that the "entropy" of a $\mathrm{BH}$ is given by, where $M$ is the mass of the $\mathrm{BH}, L_{P}$ is the Plamck length, and $A_{\text {hor }}$ is the area of the Event horizon of a black hole. This area of a $\mathrm{BH}$ event horizon is relevant since it directly connects, as we will mention later, to [2] version of super-radiance. Reference [1] version of entropy would also hold for [2] as well, and we state the entropy as

$$
S=4 \pi M^{2}=\frac{1}{4} \cdot\left(\frac{A_{\mathrm{hor}}}{L_{P}^{2}}\right) .
$$

Here, in [2] we have (27), that its main result is about the differential of the area of an event horizon which is given as follows, if there is a brane theory connection to the formation of BHs:

$$
d A_{\text {hor }}=\frac{8 \pi r_{H}}{B} d M_{\mathrm{BH}} \cdot\left(1-\frac{1}{\omega} \sum_{j=1}^{N / 2} m_{j} \cdot \Omega_{j}\right) .
$$

The positive definite nature of this expression for the differential of the area of an event horizon would then be [2] since $d M<0$, then by [2], so then by (15) below, we recover (11), by [2]; if $d A>0$, then

$$
\begin{aligned}
d M_{\mathrm{BH}} & \cdot\left(1-\frac{1}{\omega} \sum_{j=1}^{N / 2} m_{j} \cdot \Omega_{j}\right)>0 \\
& \Longleftrightarrow\left(1-\frac{1}{\omega} \sum_{j=1}^{N / 2} m_{j} \cdot \Omega_{j}\right)<0 .
\end{aligned}
$$

We make the following 3 claims as for the analogy to $\mathrm{BH}$ physics. 
Claim 1. Entropy in both Kerr and Myers-Perry BHs has $d A>0$, where $A$ is the event horizon, and

(i) for Myers-Perry BHs, the following are true (dimensions up to 10 , say, i.e., $N=10$ ):

$$
\begin{gathered}
S=4 \pi M^{2}=\frac{1}{4} \cdot\left(\frac{A_{\text {hor }}}{L_{P}^{2}}\right) \\
d A_{\text {hor }}=\frac{8 \pi r_{H}}{B} d M_{\mathrm{BH}} \cdot\left(1-\frac{1}{\omega} \sum_{j=1}^{N / 2} m_{j} \cdot \Omega_{j}\right) \\
\left(1-\frac{1}{\omega} \sum_{j=1}^{N / 2} m_{j} \cdot \Omega_{j}\right)<0 ;
\end{gathered}
$$

(ii) for Kerr BH, one could arguably have much the same thing; that is,

$$
\begin{gathered}
S=4 \pi M^{2}=\frac{1}{4} \cdot\left(\frac{A_{\text {hor }}}{L_{P}^{2}}\right) \\
d A_{\text {hor }}=\frac{8 \pi r_{H}}{B} d M_{\mathrm{BH}} \cdot\left(1-\frac{1}{\omega} m \cdot \Omega_{H}\right) \\
\left(1-\frac{1}{\omega} m \cdot \Omega_{H}\right)<0 .
\end{gathered}
$$

Proof. By (15), (9), (10), and (11), we next consider the following.

Claim 2. If $a$ is zero, then super-radiance as made possible in Claim 1 part (ii) is impossible for Kerr Black holes.

Proof. a goes to zero and mean numerator of (9) goes to zero. Hence, $\left(1-(1 / \omega) m \cdot \Omega_{H}\right)<0$ does not happen. Hence, for nonzero frequency of incoming radiation, $0<\omega<m \cdot \Omega_{H}$ does not hold. Hence, there is no BH super-radiance.

Claim 3. One could have the following: Claim 1 part (ii) may be false, but Claim 1 part (i) may be true.

Proof. For $N \geq 4$ or so, the following decomposition may be true:

$$
\left(1-\frac{1}{\omega} \sum_{j=1}^{N / 2} m_{j} \cdot \Omega_{j}\right)=\left[1-\frac{1}{\omega} m \cdot \Omega_{H}\right]-\frac{1}{\omega} \sum_{j=2}^{N / 2} m_{j} \cdot \Omega_{j}<0 .
$$

If the first term in [ ] in the RHS of the above formula is equal to 0 , Claim 1 part (ii) is false, but one could still have Claim 1 part (i) as true. That is, one could write the following.

Consider $0<\omega<\sum_{J=2}^{N / 2} m_{J} \cdot\left(\Omega_{H}\right)_{J}$. Then, Claim 1 part (i) will be true, that is, super-radiance for brane theory BHs.

The significance of the three claims is as follows. As given by [4], there is a problem, if a massive graviton exists, the bending of light, say about Mercury, the Eddinton 1919 experiment is calculated to be 3/4th the value seen in the 1919 experiment which proved classical GR. By [7], there can be a situation for which if there exists higher than 4 dimensional brane theory, one may correct the $3 / 4$ th deficiency. But if Claim 1 part (i) is not true, then the solution allowing for [7] is likely not to be true.

Note that the super-radiance phenomenon as referenced in Claim 1 part (i) and part (ii) has its roots in entropy. Note that entropy of a black hole with its surface area is stated to be a precondition for initial conditions for super-radiance. And, more than that, one needs a spinning black hole. No black hole spin, with a commensurate treatment, could lead to just black hole evaporation, as noted above, but $\mathrm{BH}$ evaporation is not the same as the super-radiance phenomenon.

3.1. Minimum Experimental Bounds Which Can Affect the Results of our Inquiry, Provided That Claim 1 Is True (as well as That Claim 3 Holds). That Is, Myers-Perry as a Higher Representation of Black Holes. Presumably Allowing Massive Gravitons. IMO, as stated above, the Meyers-Perry condition for $\mathrm{BHs}$ is, as a gateway, a probable candidate to experimental observations for BHs. As mentioned earlier, for higher dimensional BHs which may allow for massive gravitons, here are the perturbations due to GW due to higher dimensional black holes. We state these as follows.

The subsequent values by $h_{00}, h_{0 i}$, and $h_{i j}$ make the case, due to mass dependence of the black holes in the Myers-Perry black holes, has an explicit mass dependence on the mass of the black hole included. [4] has

$$
\begin{gathered}
h_{00} \approx \frac{16 \pi G}{(d-2) \cdot \Omega_{d-2}} \cdot \frac{M_{\mathrm{BH}}}{r^{d-3}}, \\
h_{i j} \approx \frac{16 \pi G}{(d-2) \cdot(d-3) \cdot \Omega_{d-2}} \cdot \frac{M_{\mathrm{BH}}}{r^{d-3}} \cdot \delta_{i j}, \\
h_{o i} \approx-\frac{8 \pi G}{\Omega_{d-2}} \cdot \frac{x^{k}}{r^{d-1}} \cdot J^{k i}
\end{gathered}
$$

The coefficient $d$ is for dimensions, 4 or above, and in this situation, with angular momentum $J^{k i}$. Here, the term put in, namely (20) is for angular area, and it has no relationship with the formula for angular velocity of BHs; namely, (20) has no relations with (4) and (9) above.

$$
\begin{gathered}
\Omega_{d-2}=\frac{2 \pi^{(d-1) / 2}}{\Gamma((d-1) / 2)}, \\
J^{k i}=2 \cdot \int x^{k} \cdot T^{i 0} \cdot d^{d-1} x .
\end{gathered}
$$

The $T^{i 0}$ above is a stress energy tensor as part of a $d$ dimensional Einstein equation given in [5] as

$$
R_{j l}-\frac{1}{2} \cdot g_{j l} \cdot R=8 \pi G T^{j l}
$$

Also, the mass of the black hole is, in this situation scaled as follows: if $\mu$ is a rescaled mass term [5],

$$
M_{\mathrm{BH}}=\mu \cdot \Omega_{d-2} \cdot \frac{(d-2)}{16 \pi G} .
$$


More generally, the mass of the black hole is written as

$$
M_{\mathrm{BH}} \equiv \int T_{00} d^{d-1} x
$$

We will next go to the minimum size of a black hole which would survive as up to 13.6 billion years and then say something about the relative magnitude of the terms in (22) and then their survival today. The variance of black hole masses, from super massive $\mathrm{BH}$ to those smaller than $10^{15}$ grams will be discussed, in the context of (19), and stress strength, with commentary as to what we referred to earlier, namely, strain for detecting GW, is given by $h(t)$ given below, with $D^{i j}$ as the detector tensor, that is, a constant term, so that, by [4, page 336$]$, we write

$$
h(t)=D^{i j} h_{i j}
$$

Equation (25) means that the magnitude of strain, $h$, is effected by (19), (20), and (21) and its magnitude, seen next. Note that the magnitude of the strain, $h$, as being brought up, may be affected by the mass of a graviton, due to $T$, which is a feed into (19) above. Namely, consider that the mass assumed for the graviton is of the order of $10^{-65}$ grams, which is given by [5]; if $h$ does not equal zero, then the stress energy tensor of the massive graviton is for nonzero $T_{u v}$ which corresponds to a nonzero concentration in interstellar space, with [5]

$$
\begin{gathered}
m_{g}^{2}=-\frac{\kappa}{6 h} T \\
T=\operatorname{trace} T_{u v} .
\end{gathered}
$$

We will get explicit upper bounds to (26) and use them as commentary in the conclusion of this paper. That will affect the infalling frequency $\omega$ which will be part of the superradiance discussion.

3.2. Values of the Meyers-Perry $h_{00}, h_{0 i}$, and $h_{i j}$ in Magnitude Lead to Nominal $h$ Values. If $D$ below is redshift corrected distance, in a rough sense, it leads to an approximation of $h$ as roughly proportional to $h_{00}$ with the roughly scaled results of

$$
h \sim \frac{G M}{c^{2} D} .
$$

Note that the tensor $D^{i j}$ is approximately unity, with the results as given by

$$
\begin{array}{r}
\left.M_{\mathrm{BH}}\right|_{\text {min-life.time }} \propto 10^{15} \text { grams } \Longleftrightarrow h_{00}, h_{i i} \propto 10^{-40} \\
\text { for BHs; } Z \text { (redshift) } \sim 10 .
\end{array}
$$

whereas super massive black holes of about 100 times the mass of our sun, at Z(redshift) of about 10 lead to significantly larger values of $h_{i i}$ as seen below,

$$
\left.M_{\mathrm{BH}}\right|_{100 \text {-solar-mass }} \Longleftrightarrow h_{00}, h_{i i} \propto 10^{-20} .
$$

It is easy from inspection to infer from this that the most early formed black holes would not be accessible and that only the giant ones would do. With that, we next then explore the frequency ranges which could lead to certain Graviton masses, as could be linked to super-radiance. That is, it would mean that a very large SMBH, of about 100 solar masses of a redshift of the order of $Z \sim 10$ at or less than a billion years after the creation of the universe, would lead to the values of (29) above, which could be conceivably detected, which then leads us to the question of what frequencies of the graviton, if presumably massive, would be involved. This would then allow us to make inquiry as to what the Meyers-Perry values for super-radiance and absorption/subsequent reflection of GW radiation which could conceivably be detected for strain values of the order given by (29) above.

3.3. Frequency and Wavelengths for Ultra Low "Massive Graviton" Masses. To get the appropriate estimates, we turn to [11], by Goldhaber and Nieta, which can be used to give a set of frequency and mass equivalences for the "massive" graviton; on the order of having the following equivalent values as paired together, namely, starting off, with graviton mass, graviton wavelength, and resulting graviton frequency, we observe the dual pairing of the following, if one also looks at Valev's estimates [12],

$$
\begin{aligned}
m_{g} & \sim 2 \times 10^{-65} \text { grams } \lambda_{g} \\
& \sim 2 \times 10^{22} \text { meters } \\
& \sim 10^{-4} \cdot \text { radius-of-universe } \omega_{g} \\
& \sim\left(\frac{3}{2}\right) \times 10^{-14} / \text { second. }
\end{aligned}
$$

Obviously, with regard to this, if such an extremely low value for resultant frequency is obtained, and then one is obtaining the value that is inevitable, just in terms of frequency, to have for any spinning Kerr BH,

$$
\omega_{g} \sim\left(\frac{3}{2}\right) \times 10^{-14} / \text { second }<\Omega_{H} .
$$

If we evaluate further for any reasonable value of $a$, we will find that, for a SMBH of about 100 solar masses, one will still have, realistically, $\omega_{g} \sim(3 / 2) \times 10^{-14} /$ second $\ll \Omega_{H}$. The author has found that for supermassive black holes for masses up to a million times the mass of the sun, the freauency the becomes, $\omega_{g} \sim(3 / 2) \times 10^{-14} /$ second $\leq \Omega_{H}$ which leads to Claim 4.

Claim 4. For super-radiance (Kerr style), $\omega_{g} \sim(3 / 2) \times$ $10^{-14} /$ second $\ll \Omega_{H}$ (easy super-radiance), for $\mathrm{SMBH}$ 100 times solar mass and $\omega_{g} \sim(3 / 2) \times 10^{-14} /$ second $\leq$ $\Omega_{H}$ (problematic super-radiance), for SMBH $10^{6}$ times solar mass.

The proof is in the definition of $\Omega_{H}=\left.\left(a /\left(2 M_{\mathrm{BH}} \cdot r_{H}\right)\right)\right|_{\text {Kerr-BH}}$, with a very small numerator.

Claim 4 means that, before the formation of massive spiral galaxies, the super-radiance is doable. However, the author fails to understand how it is possible on another 
theoretical ground; that is, what does super-radiance mean for BHs for which

$$
\begin{aligned}
\lambda_{g} & \sim 2 \times 10^{22} \text { meters } \\
& \sim 10^{-4} \cdot \text { radius-of-universe. }
\end{aligned}
$$

On the face of it, this is absurd. That is, how could wavelengths $1 / 10,000$ the size of the universe interact with a Kerr Black hole?

We claim that the embedding of black holes in five or higher dimensional space time is a way to make a connection with a multiverse, as given in the following supposition [13], and that this may be the only way to reconcile what seems to be an absurd proposition. That is, graviton wavelengths $1 / 10,000$ the size of the standard 4 dimensional universe is interacting with spinning black holes in 4 dimensional spacetime, whereas that moderate 100 times the mass of the sun BHs easily satisfy $\omega_{g} \sim(3 / 2) \times 10^{-14} /$ second $\ll \Omega_{H}$.

\section{Conclusion}

In one way, it is ridiculously easy to obtain super-radiance for massive gravitons, and, in another sense, it is an absurd proposition. Could a multiverse embedding of BHs be a way out of what otherwise seems an impossible Dichotomy? Or will we have to embrace Hawkings' suggestion that the event Horizon has foundationally crippling flaws?

To address this problem, the author looks at two suggestions. Either that the $\mathrm{BH}$ is really embedded in a multiverse and has a different geometry in higher dimensions than is supposed, or one goes to the recent Hawkings' hypothesis which changes entirely the supposition of the event horizon. Namely

We will first of all give a brief introduction to the Penrose CCC hypothesis generalized to a multiverse.

4.1. Extending Penrose's Suggestion of Cyclic Universes, Black Hole Evaporation, and the Embedding Structure our Universe Is Contained within, That Is, Using the Implications of (32) for a Multiverse. This Multiverse Embeds BHs and May Resolve What Appears to Be an Impossible Dichotomy. There are no fewer than $N$ universes undergoing Penrose "infinite expansion" (Penrose, 2006) [13] contained in a mega universe structure. Furthermore, each of the $N$ universes has black hole evaporation, with the Hawkings radiation from decaying black holes. If each of the $N$ universes is defined by a partition function, called $\left\{\Xi_{i}\right\}_{i \equiv N}^{i \equiv 1}$, then there exists information ensemble of mixed minimum information correlated as about $10^{7}-10^{8}$ bits of information per partition function in the set $\left.\left\{\Xi_{i}\right\}_{i \equiv N}^{i \equiv 1}\right|_{\text {before }}$, so minimum information is conserved between a set of partition functions per universe. Consider

$$
\left.\left.\left\{\Xi_{i}\right\}_{i \equiv N}^{i \equiv 1}\right|_{\text {before }} \equiv\left\{\Xi_{i}\right\}_{i \equiv N}^{i \equiv 1}\right|_{\text {after }} .
$$

However, there is nonuniqueness of information put into each partition function $\left\{\Xi_{i}\right\}_{i \equiv N}^{i \equiv 1}$. Furthermore, Hawkings' radiation from the black holes is collated via a strange attractor collection in the mega universe structure to form a new big bang for each of the $N$ universes represented by $\left\{\Xi_{i}\right\}_{i \equiv N}^{i \equiv 1}$. Verification of this mega structure compression and expansion of information with nonuniqueness of information placed in each of the $N$ universes favors ergodic mixing treatments of initial values for each of $N$ universes expanding from a singularity beginning. The $n_{f}$ value, will be using $(\mathrm{Ng}$, 2008) $S_{\text {entropy }} \sim n_{f}$. [14]. How to tie in this energy expression, as in (33), will be to look at the formation of a nontrivial gravitational measure as a new big bang for each of the $N$ universes as by $n\left(E_{i}\right)$. The density of states at a given energy $E_{i}$ for a partition function (Poplawski, 2011) [15]. Consider

$$
\left\{\Xi_{i}\right\}_{i \equiv 1}^{i \equiv N} \propto\left\{\int_{0}^{\infty} d E_{i} \cdot n\left(E_{i}\right) \cdot e^{-E_{i}}\right\}_{i \equiv 1}^{i \equiv N} .
$$

Each of $E_{i}$, identified with (34) above, is with the iteration for $N$ universes $(\mathrm{Ng}, 2008)$ [14]. Then the following claim holds.

Claim 5. Consider

$$
\begin{aligned}
\left.\frac{1}{N} \cdot \sum_{j=1}^{N} \Xi_{j}\right|_{j \text {-before-nucleation-regime }} & \left.\underset{\text { vacuum-nucleation-tranfer }}{\longrightarrow} \Xi_{i}\right|_{i \text {-fixed-after-nucleation-regime }}
\end{aligned}
$$

For $N$ number of universes, with each $\left.\Xi_{j}\right|_{j \text {-before-nucleation-regime }}$ for $j=1$ to $N$ being the partition function of each universe just before the blend into the RHS of (35) above for our present universe. Also, each of the independent universes given by $\left.\Xi_{j}\right|_{j \text {-before-nucleation-regime }}$ are constructed by the absorption of one to ten million black holes taking in energy. That is, $(\mathrm{Ng}, 2008)$ [14]. Furthermore, the main point is similar to what was done in [16] in terms of general ergodic mixing.

\section{Claim 6. Consider}

$$
\left.\left.\Xi_{j}\right|_{j \text {-before-nucleation-regime }} \approx \sum_{k=1}^{\operatorname{Max}} \widetilde{\Xi}_{k}\right|_{\text {black-holes-jth-universe }} \cdot
$$

What is done in Claims 5 and 6 is to come up with a protocol as to how a multidimensional representation of black hole physics enables continual mixing of space and time [17] largely as a way to avoid the Anthropic principle, as to a preferred set of initial conditions. With investigations, this complex multiverse may allow bridging what seems to be an unworkable dichotomy between ultralow graviton frequency, corresponding roughly to $10^{-65}$ grams in rest mass, easily satisfied by Kerr black holes with rotational frequencies, as given in our text as many times greater, combined with the absurdity of what (32) is. How can a graviton with a wavelength $10^{-4}$ the size of the universe interact with a Kere black hole, spatially? Embedding the $\mathrm{BH}$ in a multiverse setting may be the only way out.

Claim 5 is particularly important. The idea here is to use what is known as CCC cosmology, which can be thought of as follows. 
First. Have a big bang (initial expansion) for the universe. After redshift $z=10$, a billion years ago, $\mathrm{SMBH}$ formation starts. Matter-energy is vacuumed up by the SMBHs, which at a much later date than today (present era) gather up all the matter-energy of the universe and recycle it in a cyclic conformal translation as follows:

$$
E=8 \pi \cdot T+\Lambda \cdot g
$$

$$
\begin{aligned}
& E=\text { source for gravitational field } \\
& T=\text { mass energy density } \\
& g=\text { gravitational metric } \\
& \Lambda=\text { vacuum energy, rescaled as follows }
\end{aligned}
$$

$$
\Lambda=c_{1} \cdot[\mathrm{Temp}]^{\beta},
$$

where $c_{1}$ is a constant. Then

The main methodology in the Penrose proposal has been in (38) evaluating a change in the metric $g_{a b}$ by a conformal mapping $\widehat{\Omega}$ to

$$
\widehat{g}_{a b}=\widehat{\Omega}^{2} g_{a b} .
$$

Penrose's suggestion has been to utilize the following [18]

$$
\widehat{\Omega} \underset{\mathrm{ccc}}{\longrightarrow} \widehat{\Omega}^{-1} \text {. }
$$

Infall into cosmic black hopes has been the main mechanism which the author asserts would be useful for the recycling apparent in (40) above with the caveat that $\hbar$ is kept constant from cycle to cycle as represented by

$$
\hbar_{\text {old-cosmology-cycle }}=\hbar_{\text {present-cosmology-cycle }} \text {. }
$$

Equation (40) is to be generalized, as given by a weighing averaging as given by (35) where the averaging is collated over perhaps thousands of universes, call that number $N$, with an ergodic mixing of all these universes, with the ergodic mixing represented by (35) to generalize (40) from cycle to cycle.

4.2. Conclusion, Future Prospects. If this does not work, and the multiverse suggestion is unworkable, there, then, has to be a consideration of the zero option, namely, Hawkings throwing out the event horizon as we know it in $\mathrm{BH}$ physics. See this reference, namely, [16].

We are in for interesting times. I see turbulence and interesting results ahead.

\section{Conflict of Interests}

The author declares that there is no conflict of interests regarding the publication of this paper.

\section{Acknowledgment}

This work is supported in part by the National Nature Science Foundation of China Grant no. 11375279.

\section{References}

[1] T. Padmanabhan, Gravitation, Foundations and Frontiers, Cambridge University Press, New York, NY, USA, 2010.

[2] E. Jung, S. H. Kim, and D. K. Park, "Condition for the superradiance modes in higher-dimensional rotating black holes with multiple angular momentum parameters," Physics Letters B, vol. 619, no. 3-4, pp. 347-351, 2005.

[3] S. Weinberg, Gravitation and Cosmology: Principles and Applications of the General Theory of Relativity, Wiley Scientific, New York, NY, USA, 1972.

[4] P. Tinyakov, "Giving mass to the graviton," in Particle Physics, and Cosmology, the Fabric of Space-Time, F. Bernadeau, C. Grojean, and J. Dalibard, Eds., pp. 471-499, Elsevier, Oxford, UK, 2007, part of Les Houches, Session 86.

[5] M. Maggiore, Gravitational Waves: Volume 1. Theory and Practice, Oxford University press, Oxford, UK, 2008.

[6] P. Creminelli, A. Nicholis, M. Papucci, and E. Trincherini, "Ghosts in massive gravity," Journal of High Energy Physics, vol. 2005, Article ID 0509, 2005.

[7] C. Csáki, J. Erlich, and T. J. Hollowood, “Graviton propagators, brane bending and bending of light in theories with quasilocalized gravity," Physics Letters B, vol. 481, no. 1, pp. 107-113, 2000.

[8] M. Meyers, "Meyers-Perry Black holes," in Black Holes in Higher Dimensions, G. Horowitz, Ed., pp. 101-131, Cambridge University Press, New York, NY, USA, 2012.

[9] Black Holes in Higher Dimensions, G. Horowitz, Ed., Cambridge University Press, New York, NY, USA, 2012.

[10] M. Porrati, "Fully covariant van Dam-Veltman-Zakharov discontinuity, and absence thereof," Physics Letters B, vol. 534, no. 1-4, pp. 209-215, 2002.

[11] A. S. Goldhaber and M. M. Nieta, "Photon and graviton mass limits," Reviews of Modern Physics, vol. 82, pp. 939-979, 2010.

[12] D. Valev, "Phenominological mass relationship for free massive stable particles and estimations of neutrino and graviton mass," http://arxiv.org/pdf/1004.2449vl.pdf.

[13] R. Penrose, "Before the big bang. An outrageous new perspective and its implications for particle physics," in Proceedings of European Particle Accelerator Conference (EPAC '06), pp. 27592763, 2006

[14] Y. J. Ng, "Spacetime foam: from entropy and holography to infinite statistics and nonlocality," Entropy, vol. 10, no. 4, pp. 441-461, 2008.

[15] N. Poplawski, "Cosmological constant from QCD vacuum and torsion," Annalen der Physik, vol. 523, pp. 291-295, 2011.

[16] S. W. Hawkings, "Informational preservation and weather forecasting for black holes," http://arxiv.org/abs/1401.5761.

[17] H. A. Dye, "On the ergodic mixing theorem," Transactions of the American Mathematical Society, vol. 118, pp. 123-130, 1965.

[18] R. Penrose, Cycles of Time-An Extrardinary New View of the Universe, Alfred A. Knopf, New York, NY, USA, 2011. 

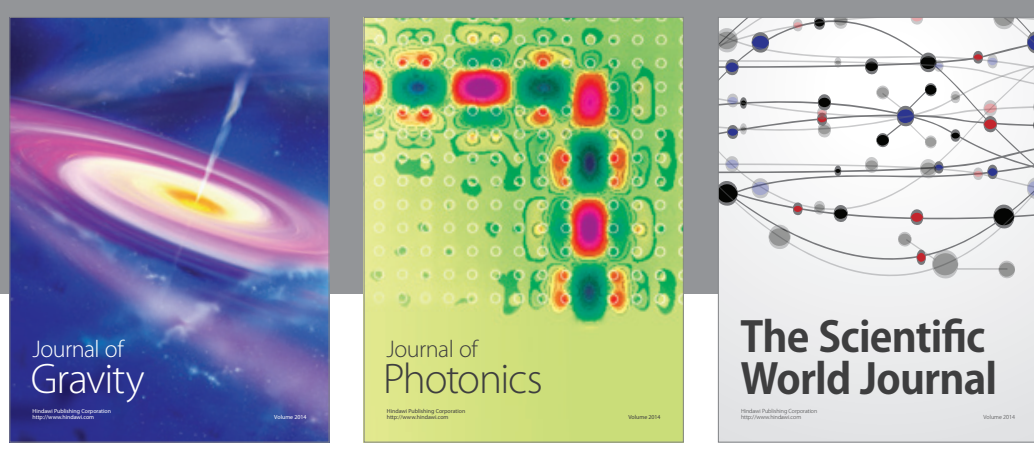

The Scientific World Journal
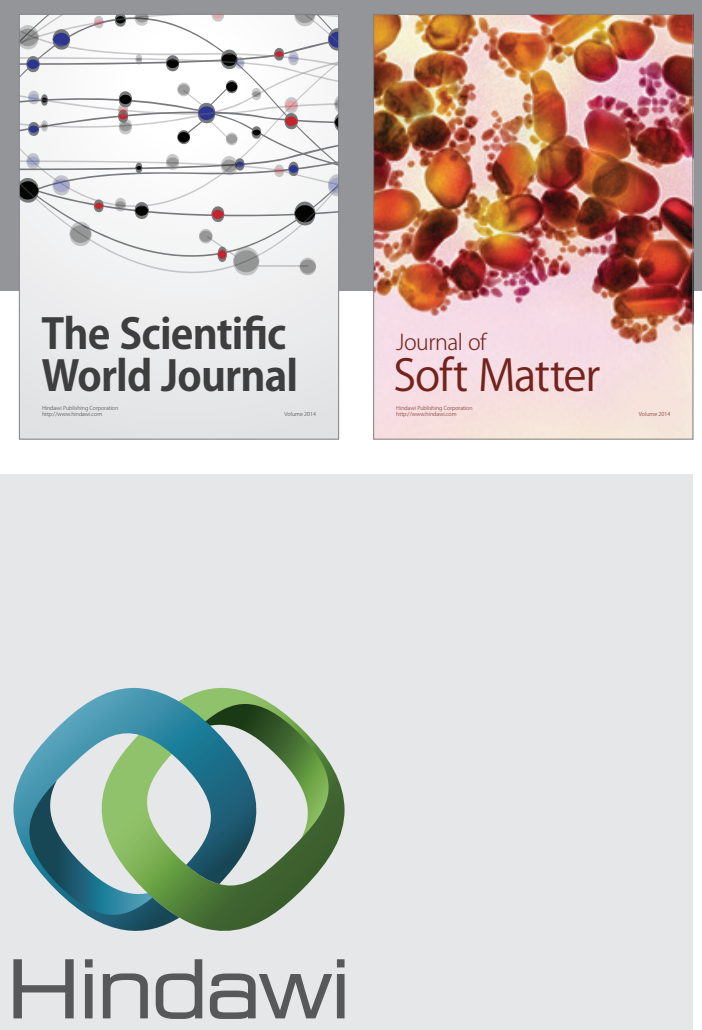

Submit your manuscripts at

http://www.hindawi.com

nternational Journal of

Statistical Mechanics
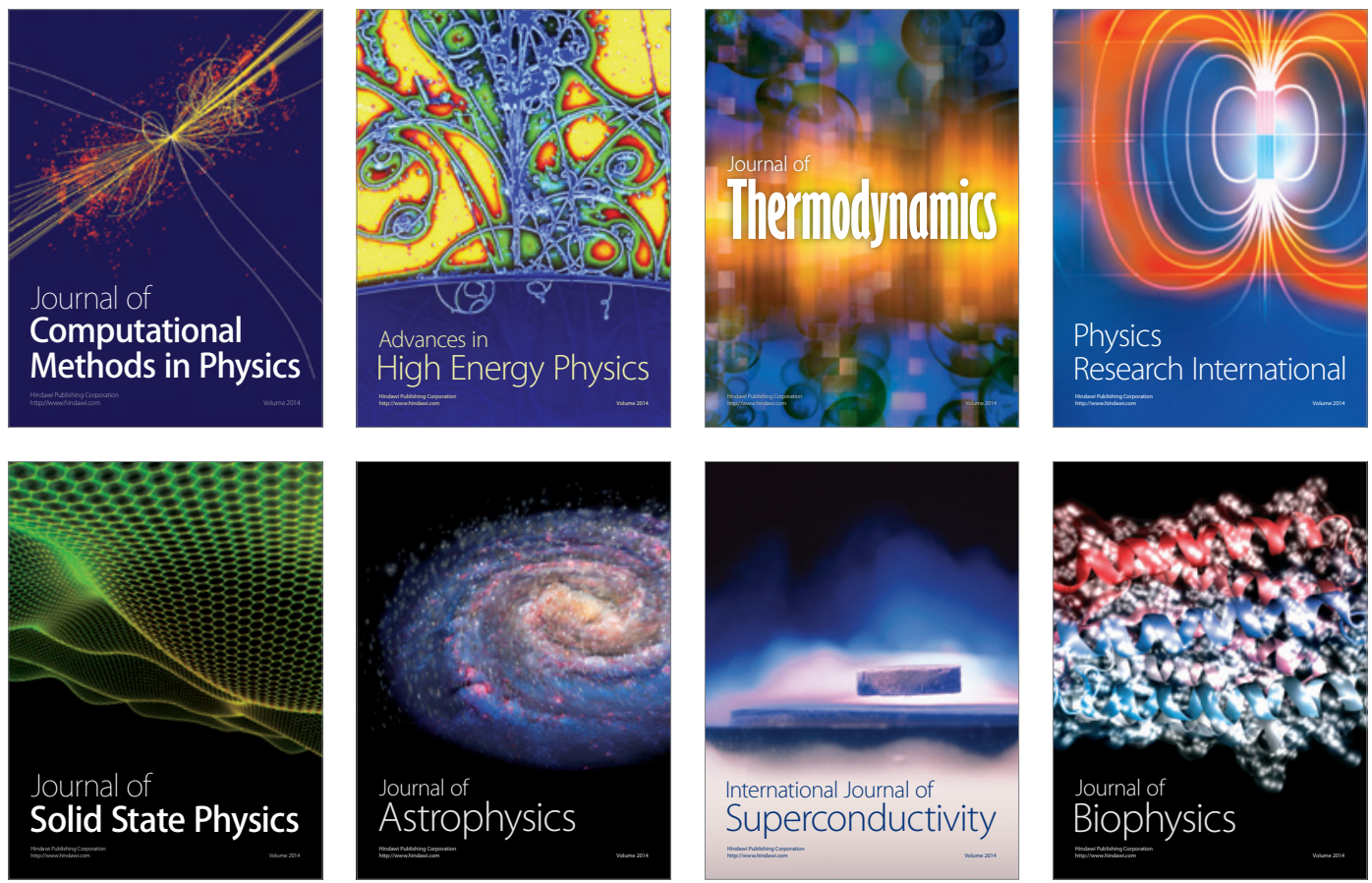
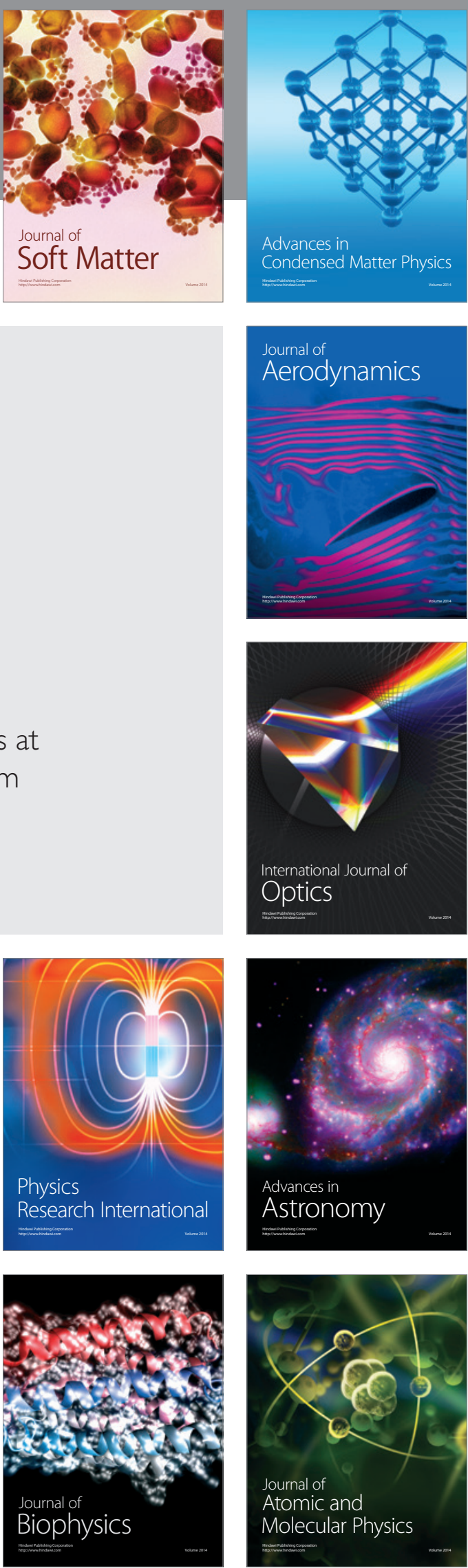